\title{
Biofilm Genes among Drug Resistant Pseudomonas Aeruginosa
}

\section{http://bjas.journals.ekb.eg}

\section{S.A.Samie ${ }^{1}$, Y.E. Rizk ${ }^{2}$, N.F.Al-Husseini ${ }^{3}$ and D.A.Ibrahim}

${ }^{1}$ Chemical Pathology Dept., Faculty of Medicine, Benha Univ., Benha, Egypt

${ }^{2}$ Cardio-Thorathic Surgery Dept., Faculty of Medicine, Benha Univ., Benha, Egypt

${ }^{3}$ Medical Biochemistry Dept., Faculty of Medicine, Benha Univ., Benha, Egypt

E-Mail: ${ }^{1}$ D.A.I356@gmail.com

\begin{abstract}
P. aeriogenosa is an opportunistic pathogen frequently associated to nosocomial infections, especially in immunocompromised hosts.Biofilm production is an important mechanism for the survival of Pseudomonas aeruginosa and its relationship with antimicrobial resistance represents a challenge for patient therapeutics. Pseudomonas aeruginosa is an opportunistic pathogen frequently associated to nosocomial infections, especially in immunocompromised hosts. The aim of this study to assess the role of some selected genes and the formation of biofilm in clinically isolates of drug resistant Pseudomonas aeruginosa.This study included 35 patients who were admitted in different inpatient departments at a Benha University Hospital and Benha Children Hospital during the period from August 2018 to December 2019.The samples were tips of indwelling medical device and urine samples were taken from patients using urinary catheters. These isolates were tested from antibiotic sensitivity by disk diffusion method and were screened phenotypically for biofilm formation by Congo Red Agar (CRA) method and Tissue Culture Plate (TCP) technique .The antibiogram of all isolates was determined and reveald that (XDR were 54.3\%), (MDR were 8.6\%)and (Non MDR/XDR were 37.1\%). The presence of RhlI and PelB genes in biofilm forming P. aeruginosa isolates was tested by using polymerase chain reaction (PCR).The results revealed the presence of these genes in both strong and weak biofilm producer isolates.These final results showed the significance of these genes in biofilm formation and suggest the presence of other factors which may contribute in determining the degree of biofilm formation in P. aeriogenosa.
\end{abstract}

Keywords : Biofilm Formation, Pseudomonas aeruginosa, Genes -Medical device.

\section{Introduction}

Pseudomonas aeruginosa is most frequently associated with human infections. The bacterium is regarded as an opportunistic pathogen, primarily causing nosocomial infections in immunocompromised patients [1].

Biofilm-forming bacteria are often seen on the surfaces of tissues and biomaterials at sites of persistent infection. Medical implants and catheters are particularly susceptible to biofilm formation because immune responses are significantly reduced in proximity to foreign bodies .In fact, biofilm formation is a main cause of implant failure and often limits the lifetime of many indwelling medical devices [2].

In addition, bacterial populations in biofilms are considerably more resistant than free-living planktonic cells are to antibacterial agents [3].

P. aeruginosa has two hierarchical QS systems known as las and rhl It has been repeatedly reported that one rhlcontrolled factor has multiple functions in biofilm development [4].

Pseudomonas aeruginosa causes chronic biofilm infections and has the capacity to synthesize three exopolysaccharides implicated in biofilm formation: alginate, the polysaccharide synthesis locus (PSL), and pellicle (PEL) polysaccharides [5].

The role of Pel in biofilm formation was first identified in a screen for mutants deficient in pellicle formation (i.e., biofilms forming at the air-liquid interface of standing cultures) Pel was later shown to be important for initiating and maintaining cell-cell interactions in biofilms [6].In this study, we aimed to to assess the role of some selected genes and the formation of biofilm in clinically isolates of drug resistant Pseudomonas aeriogenosa.

\section{Methodology}

\section{A. Patients and isolates}

In the present study, thirty five Pseudomonas aeruginosa clinical isolates were collected from patients who were admitted in different inpatient departments at a Benha University Hospital and Benha Children Hospital. This study was carried out in Microbiology unit, Clinical Pathology Departments at Benha university hospitals during the period from August 2018 to December 2019.

The samples were tips of indwelling medical device, after it had been removed from the patients and their types and numbers as follows :central venous catheters 19(54.3\%), peripheral catheter $3(8.6 \%)$, synthetic vascular grafts 5( $14.3 \%$ ) and urine samples were taken from patients using urinary catheters $8(22.9 \%)$.

These isolates were identified depending on cultures characters of colonies on MacConkey agar, pigment production on nutrient agar plates. Preliminary biochemical identification of isolates was done by using oxidase test, citrate utilization test, motility test, ornithine decarboxylation test, urease test and reaction on triple sugar iron agar and growth at $42 \mathrm{C}^{\circ}$.Identification of Pseudomonas spp. was performed according to [7].

\section{B. Antimicrobial susceptibility testing}

The antimicrobial susceptibility testing of the planktonic cells of all isolates towards amikacin, cefepime, ceftazidime, tobramycin, gentamicin, imipenem, colistin, ciprofloxacin, piperacillin- tazobactam and polymyxin B were 
determined by the disk diffusion method using Muller Hinton agar (Oxoid) according to the clinical laboratory standards institute $[8,9$.

Criteria for defining MDR, XDR and PDR in Pseudomonas aeruginosa [10]

- Multidrug-resistant (MDR): The isolate is nonsusceptible to at least 1 agent in $\geq 3$ antimicrobial categories used for the treatment of this organism.
- Extensively drug-resistant (XDR): The isolate is nonsusceptible to at least 1 agent in all but 2 or fewer antimicrobial categories remain susceptible.

- Pandrug-resistant (PDR): Non-susceptibility to all agents in all antimicrobial categories for each bacterium.

Table(1) Antimicrobial categories and agents used to define MDR, XDR and PDR [10].

\begin{tabular}{lc}
\hline Antimicrobial category & Antimicrobial agent \\
\hline Aminoglycosides & Gentamicin, Tobramycin, Amikacin, Netilmicin \\
Antipseudomonal carbapenems & Imipenem, Meropenem, Doripenem \\
Antipseudomonal penicillins $+\boldsymbol{\beta}$-lactamase inhibitors & Ticarcillin-clavulanic acid \\
& Piperacillin-tazobactam \\
Antipseudomonal fluoroquinolones & Ciprofloxacin, Levofloxacin \\
Extended spectrum cephalosporins & Ceftazidime, Cefepime \\
Monobactams & Aztreonam \\
Phosphonic acids & Fosfomycin \\
Polymixins & Colistin, Polymixin B \\
\hline
\end{tabular}

C. Phenotypic detection of biofilm formation by $P$. aeruginosa isolates

1- Tissue Culture Plate method (TCP) [11]

The ability of organisms to form biofilm was tested by growing them in the wells of microwell plastic plate and then measuring the optical densities after staining the wells. Organisms were isolated from fresh agar plates and were inoculated on $10 \mathrm{ml}$ of Trypticase soy broth with $1 \%$ glucose and were inoculated at $37 \mathrm{c}$ for $24 \mathrm{hr}$.The cultures were then diluted 1:100 with fresh medium. Individual well of sterile 96 well- flat bottom polystyrene tissue culture treated plates were filled with $200 \mu \mathrm{l}$ of diluted cultures.Negative control wells contained sterile broth. The plates were incubated at 37 c for $24 \mathrm{hr}$. After incubation, contents of each well were removed gently by tapping. The wells were washed with 200 $\mu 1$ of phosphate buffer saline (PH 7.2) four times. Biofilm formed by bacteria adherent to the wells were fixed by $2 \%$ sodium acetate for $20 \mathrm{~min}$ and were stained by crystal violet $(0.1 \%)$ for $15 \mathrm{~min}$.The microwell plastic plate was washed 3 times with taps water using Pasteur pipette and was left at room temperature to air dry. The Optical density (OD) of stained adherent bacteria was read on ELISA reader Diasowce (Belgium) at wave length $570 \mathrm{~nm}$ OD.The mean OD value obtained from negative control were deduced from the average test OD values to obtain the OD value of each test strain. Biofilm formation by isolates was analyzed and grouped relying on the absorbance of the crystal violetstained attached cells as weak, moderate or strong biofilm forming strainTable (2).

Table (2) Interpretation of biofilm production by TCP method [11].

\begin{tabular}{lcc}
\hline Mean OD values & Adherence & Biofilm formation \\
\hline$<0.124$ & Non & Non $/$ Weak \\
$0.120-0.240$ & Moderate & Moderate \\
$>0.240$ & Strong & Strong \\
\hline
\end{tabular}

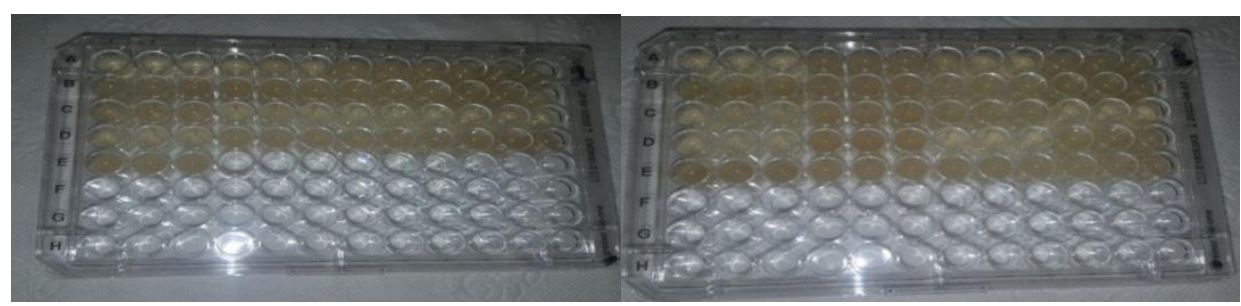

Fig (1) 2 wells of a 96 flat bottomed microwell plastic plate contain the inoculated TSB with $1 \%$ glucose) after overnight incubation at $37 \mathrm{C}^{\circ}$ 


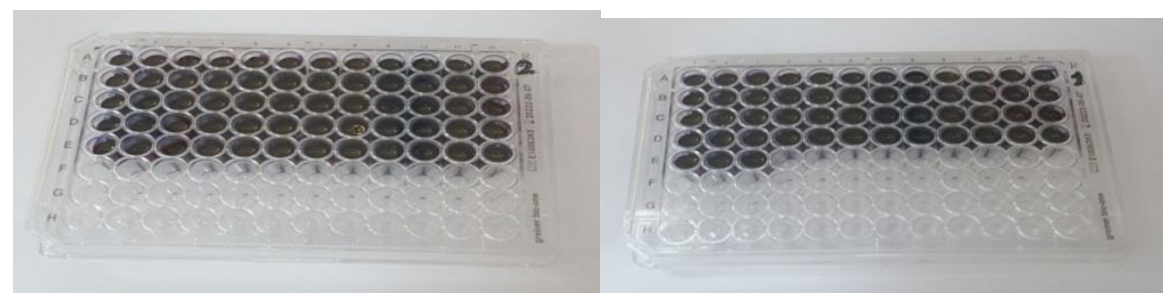

Fig (2) Crystal violet $(0.1 \%)$ was added to each well for biofilm staining.

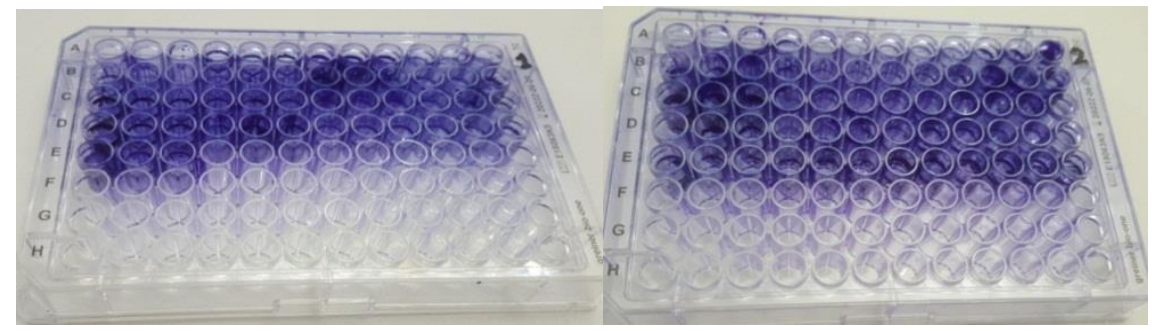

Fig (3) TPC after staining with crystal violet $0.1 \%$ Optical density (OD) of sained adherent bacteria was determined with ELISA autoreader Diasowce

(Belgium)at wave length $570 \mathrm{~nm}$.

\section{2- Congo red agar [12]}

The Congo red dye directly interacts with certain polysaccharides, forming colored complexes. Congo red has the ability to react with the slimy material giving black discoloration. Biofilm producers form black colonies on CRA, whereas non producers form red colonies [13].
CRA medium was prepard with Brain heart infusion (BHI) $(37 \mathrm{gms} / \mathrm{L})$, sucrose $(50 \mathrm{gms} / \mathrm{L})$, agar no.1 (10 gms/L) and congo red stain $(0.8 \mathrm{gms} / \mathrm{L})$. Positive result is indicated by black colonies with a dry crystalline consistency. Pink colonies are considered non slime producers [12].

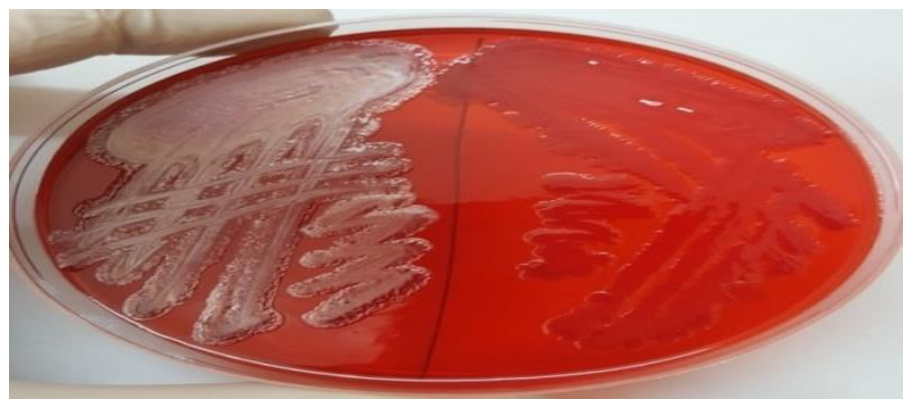

Fig (4) CRA method for detection of biofilm production.

A- Biofilm producers: black colonies B- Non biofilm producers: red colonies.

\section{Genotypic detection of genes coding for biofilm} formation(RhlI and PelB genes) by PCR

PCR is a test for in vitro amplification of specific DNA sequences using two primers that hybridize to opposite strands and flank target DNA region.

Sequences flanking RhlI and PelB polymorphism were PCR- amplified from genomic DNA using a pair of specific oligonucleotide primers, The amplified products were the analyzed by electrophoresis. The RhlI was detected as a 245bp band and the PelBwas visualized as $190 \mathrm{bp}$.
DNA extraction was done according to the manufacture's by use of G-Spin TM Total DNA Extraction Kit.

Gene amplification using polymerase chain reaction :Enzymatic amplification was performed by PCR using IStar TM Taq DNA polymerase enzyme (Intron Biotechnology ) and Vereti thermal cycler ( AB Applied Biosystem )

The reaction mixture included :Extracted DNA template,(Master Mix M-M ) 2× Easy Taq@ PCR SuperMix and Primers (Metabion International AG,Germany )

- RhlI Forward 5-CTCTCTGAATCGCTGGAAGG-3

- RhII Reverse 5-GCGAAGACTTCCTTGAGCAG-3 
- PelB Forward 5-CGCCTGCTCTGGTTCTACAT-3

- PelB Reverse 5-AGTCGTTGGGATTGGACTTG-3

Detection of PCR Amplification products using Gel Electrophoresis and Ultra- Violet Light Transillumination:The amplified samples were then run on $2 \%$ agarose gel in the presence of a DNA marker using gel electrophoresis and visulalized on a UV transilluminator to detect presence of amplified product the RhlI and PelB genes.

\section{E. Statistial analysis}

The data were recorded on an "Investigation report form". These data were tabulated, coded then analyzed using the computer program SPSS (Statistical package for social science) version 16 to obtain Descriptive data.

Descriptive statistics were calculated for the data in the form of: Mean,Standard deviation $( \pm \mathrm{SD})$ and Number and percent

In the statistical comparison between the different groups, the significance of difference was tested using one of the following tests:-Student 's t-test:-Used to compare between mean of two groups of numerical (parametric) data,For continous non- parametric data, Mann-Whitney Utest was used for inter-group analysis and Inter-group comparison of categorical data was performed by using chi square test $\left(\mathrm{X}^{2}\right.$-value), A $\mathrm{P}$ value $<0.05$ was considered statistically significant $(\mathrm{S})$. And a $\mathrm{P}$ value $<0.001$ was considered highly significant (HS) in all analyses.

\section{Results}

The study included 35 patients with 23 males $(65.7 \%)$ and 12 females $(34.3 \%)$; their age ranged from 3 to 80 years with a mean of52.54 \pm 20.05 .

The majority of Pseudomonas aeruginosa isolates were isolated from hematology department patients (37.1\%); followed by nephrology department (31.4\%); then urology department $(22.9 \%)$ and finally $(8.6 \%)$ from burn department

The highest percentage of $P$. aeruginosa isolates were from patients with neutropenic fever $(37.1 \%)$; followed by patients with infected hemodialysis chronic kidney disease (HD-CKD) $(31.4 \%)$ and patients with urosepsis (14.3\%); then from patients with urinary tract infection (UTI) $(8.6 \%$ ) and lastly $(8.6 \%)$ from patients with infected burn.

From each patient included in the study, one sample was collected. $P$. aeruginosa isolates were recovered from central venous catheters, 19 (54.3\%), urine collected from urine catheters, 8 (22.9\%), synthetic vascular grafts, 5 $(14.3 \%)$ and peripheral catheters, $3(8.6 \%)$.

\section{Antibiotic sensitivity profile of isolated Pseudomonas aeruginosa strains}

The highest resistance rate was to cefepime $(100 \%)$ followed by gentamicin and tobramycin (62.9\%), while the highest sensitivity rate was to colistin (100\%) followed by polymyxim B (97.1\%) and lastly imipeneme ( 54.3) Fig (5).

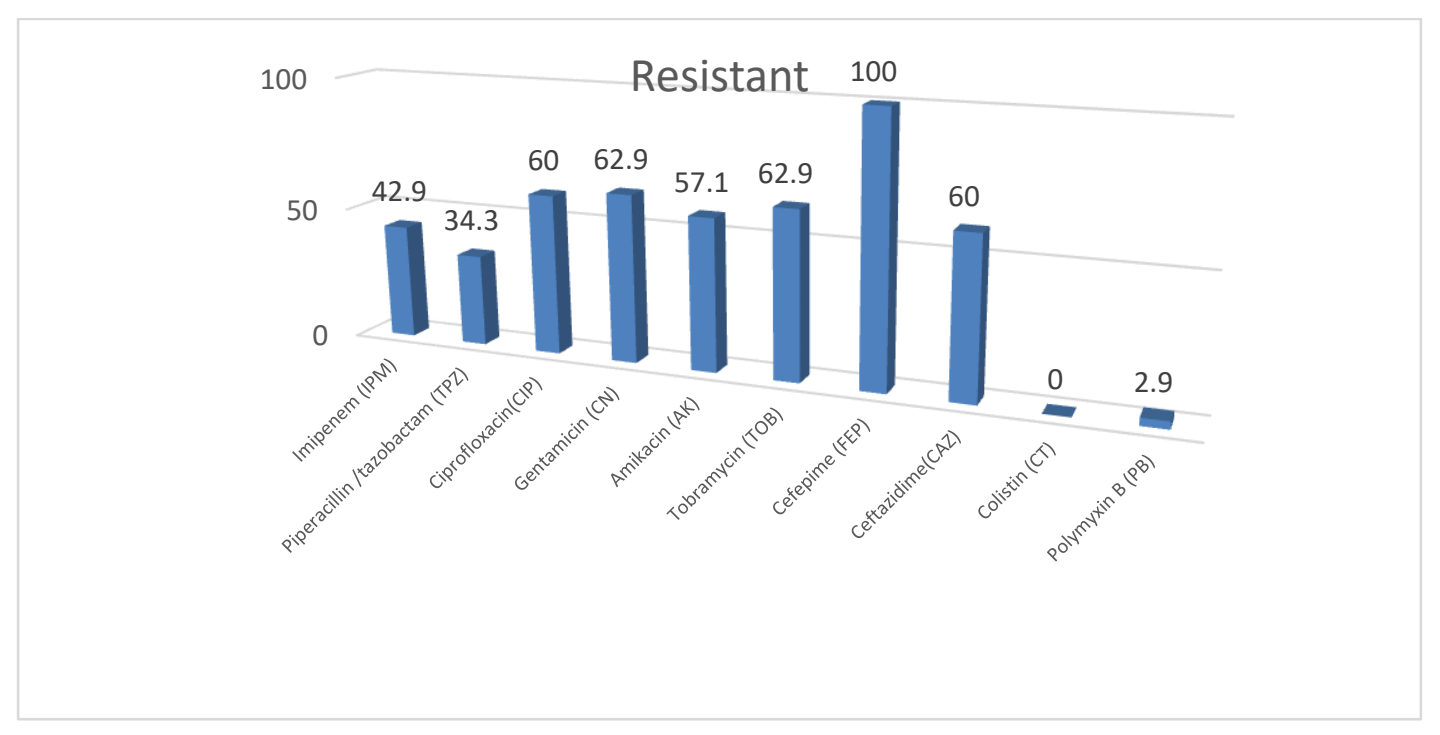

Fig (5) Percentage resistance of the P.aeruginosa isolates toward different antimicrobial agents.

Among the 35 studied P.aeruginosa isolates,XDR were detected in 19 isolates $(54.3 \%)$, while 3 isolates $(8.6 \%)$ were considered as MDR and 13 isolates $(37.1 \%)$ were Non - MDR/ XDR Fig (6,7,8 and 9). 


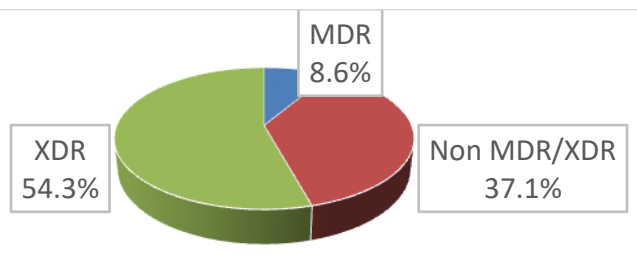

- MDR - Non MDR/XDR = XDR

Fig (6) Pattern of antimicrobial resistance among Pseudomonas aeriogenosa.

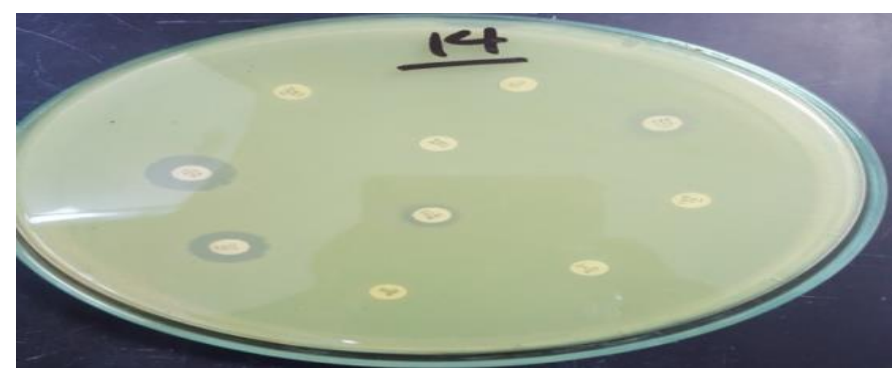

Fig (7) XDR Pseudomonas aeriogenosa isolate on included study showes resistance to all antibiotics tested except colistin and polymyxin $\mathrm{B}$.

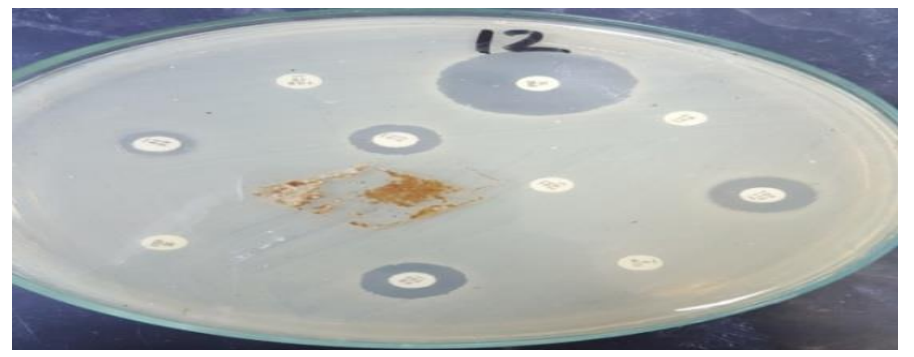

Fig (8) MDR Pseudomonas aeriogenosa on included study showes resistance to all antibiotics tested except imipenime,colistin and polymyxin B, intermediate sensitive to pepracillin tazobactam.

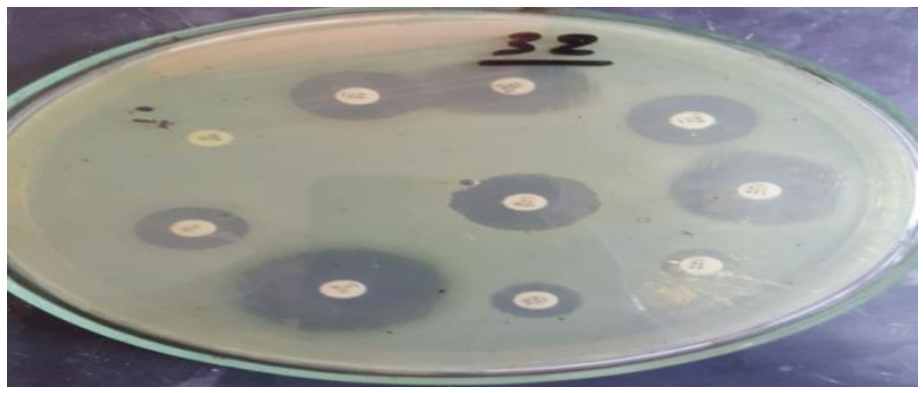

Fig (9) Non - MDR/XDR Pseudomonas aeriogenosa onincluded study showes susceptibility to all antibiotics tested except cefepime. 
Phenotypic detection of biofilm formation by $P$. aeruginosa isolates

The 35 Pseudomonas aeruginosa isolates were examined for their ability to adhere and form biofilm using: Tissue Culture Plate (TCP) method [11], and Congo red agar (CRA) method [12].
By the TCP method; 8 (22.9\%) of isolated organisms were weak biofilm producers, $11(31.4 \%)$ were moderate biofilm producers, $16(45.7 \%)$ were strong biofilm producers .However ;by CRA test $24(68.6 \%)$ of isolated organisms were biofilm producers, while $11(31.4 \%)$ were non biofilm producers Table $(3,4)$.

Table (3) Biofilm production by the TCP method.

\begin{tabular}{lcc}
\hline & No. & \% \\
\hline Weak & 8 & 22.9 \\
Moderate & 11 & 31.4 \\
Strong & 16 & 45.7 \\
Total & 35 & 100.0 \\
\hline
\end{tabular}

Table (4) Biofilm production by the CRA method.

\begin{tabular}{lcc}
\hline & No. & $\%$ \\
\hline Non biofilm forming & 11 & 31.4 \\
Biofilm forming & 24 & 68.6 \\
Total & 35 & 100.0 \\
\hline
\end{tabular}

Impact of biofilm formation on antibiotic resistance

Antimicrobial susceptibility profile of Pseudomonas aeruginosa regarding biofilm production by the CRA method: All biofilm forming isolates were resistant to cefepime $(100 \%$ ) followed by both gentamicin and tobramycin $(79.2 \%)$ and followed by both amikacin and ceftazidim $(75 \%)$.

Antimicrobial susceptibility profile of Pseudomonas aeruginosa regarding biofilm production by the TCP method: All strong biofilm forming isolates were resistant to cefepime $(100 \%)$ followed by both gentamicin and tobramycin $(75 \%)$ and followed by ciprofloxacin, amikacin and ceftazidim $(68.8 \%)$. All moderate biofilm forming isolates were resistant to cefepime $(100 \%)$ followed by both ciprofloxacin gentamicin,amikacin and tobramycin $(63.6 \%)$ and followed by imipenem $(54.5 \%)$.All weak biofilm forming isolates were resistant to cefepime (100\%) followed by ciprofloxacin,gentamicin, tobramycin and ceftazidem $(37.5 \%)$.

As regards the relation between biofilm grading and antibiotic resistance, it was observed that the moderate biofilm - forming isolates were XDR (31.6\%) compared to the strong biofilm forming $(52.6 \%)$. Where as the moderate biofilm forming isolates were MDR $(66.7 \%)$ compared to the strong biofilm forming( $33.3 \%)$. There is no significant relationship between pattern of drug resistance and biofilm grading in Pseudomonas aeruginosa Table (5).

Table (5) Correlation between pattern of drug resistance and biofilm grading in Pseudomonas aeruginosa.

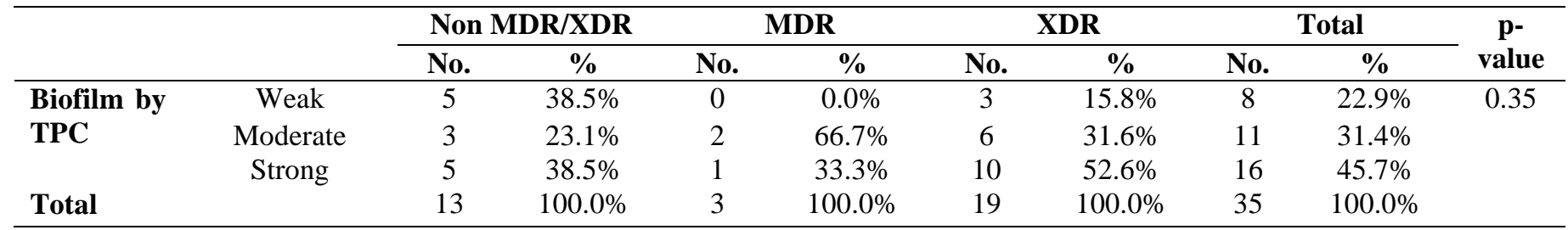

Detection of RhlI and PelB genes by PCR

PCR screening for the specific sequences of RhlI gene showed a PCR product at $245 \mathrm{bp}$, as shown in Fig ( $10,11,12,13,14,15,16)$. This quorum sensing gene was detected in all screened isolates including weak, moderate and strong biofilm forming isolates.

Regarding PelB gene detection, a PCR product was observed at 190 bp in its PCR amplification as shown in Fig
$(10,11,12,13,14,15,16)$. This gene (exopolysaccharide encoding gene) was detected in all screened isolates including weak, moderate and strong biofilm forming isolates.

\section{Detection of RhII and PelB genes by PCR}




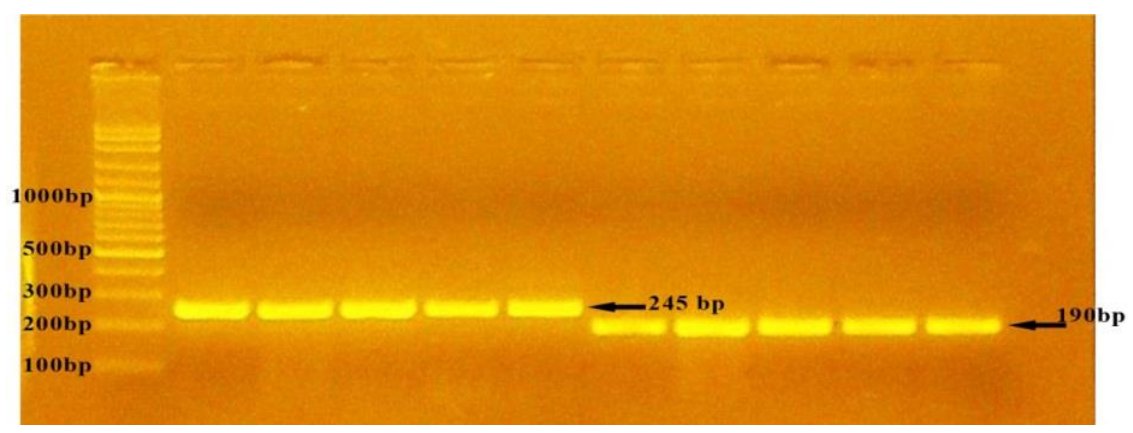

Fig (10) Agarose gel electrophoresis of RhlI gene amplicons(245 bp) andPelB gene amplicons (190 bp). MWM-molecular weight marker (100 bp).Lanes from 1 to 5 were ampliconsof RhlI gene from isolates NO 1,2,3,4,5. Lanes from 6 to 10 were amplicons of PelB gene from isolates NO 1,2,3,4,5.

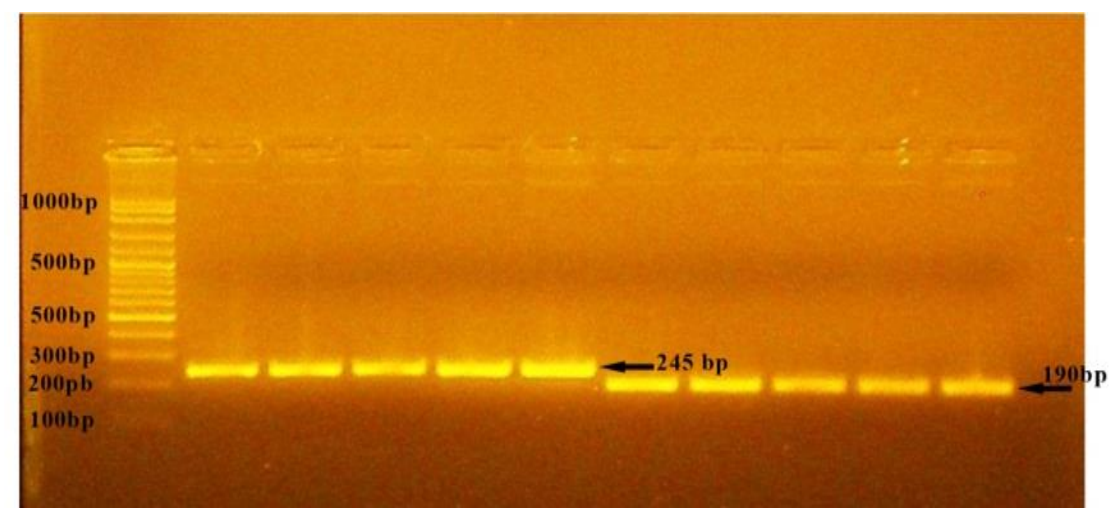

Fig (11) Agarose gel electrophoresis of RhlI gene amplicons(245 bp) andPelB gene amplicons (190 bp). MWM-molecular weight marker (100 bp).Lanes from 1 to 5 were ampliconsof RhlI gene from isolates NO. $6,7,8,9,10$. Lanes from 6 to 10 were amplicons of PelB gene from isolates $\mathrm{NO} 6,7,8,9,10$.

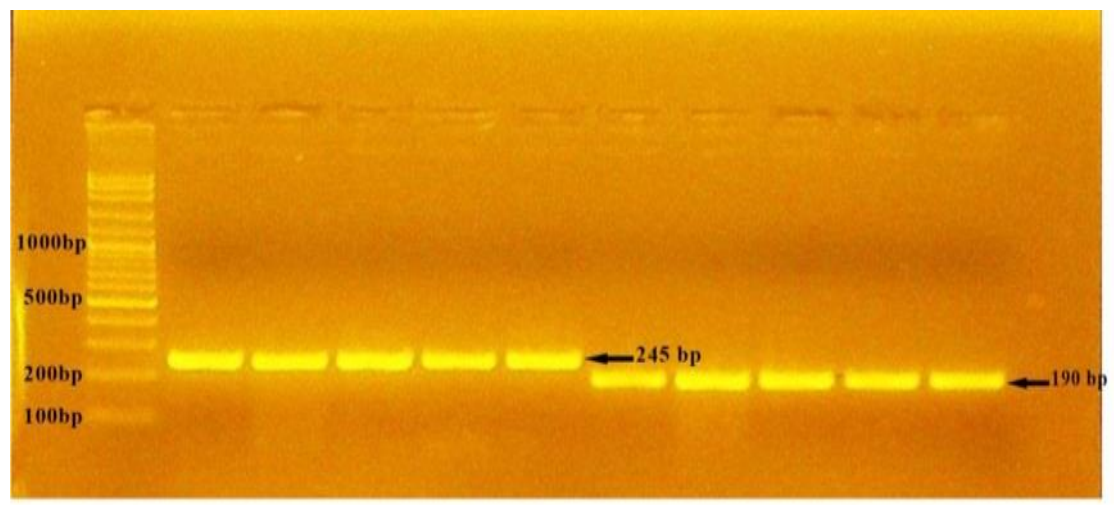

Fig (12) Agarose gel electrophoresis of RhlI gene amplicons(245 bp) andPelB gene amplicons (190 bp). MWM-molecular weight marker (100 bp).Lanes from 1 to 5 were ampliconsof RhlI gene from isolates NO. $11,12,13,14,15$. Lanes from 6 to 10 were amplicons of PelB gene from isolates NO 11,12,13,14,15. 


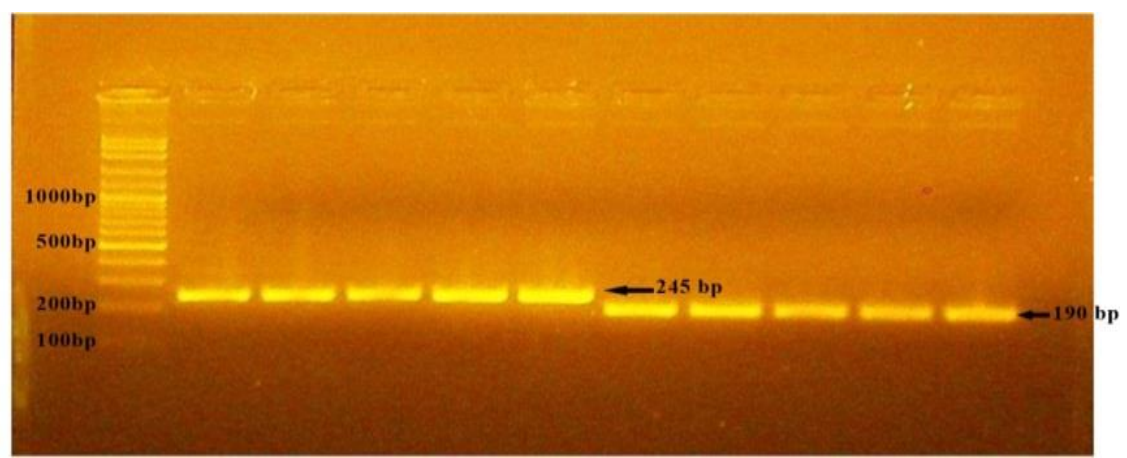

Fig (13) Agarose gel electrophoresis of RhlI gene amplicons(245 bp) andPelB gene amplicons(190 bp). MWM-molecular weight marker (100 bp).Lanes from 1 to 5 were ampliconsof RhlI gene from isolates NO 16,17,18,19,20. Lanes from 6 to 10 were amplicons of PelB gene from isolates $16,17,18,19,20$.

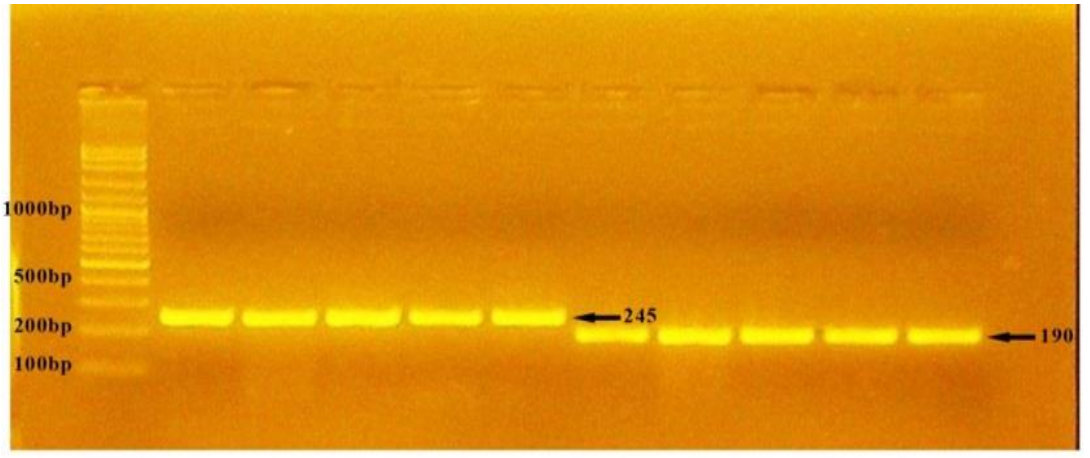

Fig (14) Agarose gel electrophoresis of RhlI gene amplicons(245 bp) andPelB gene amplicons (190 bp). MWM-molecular weight marker (100 bp).Lanes from 1 to 5 were ampliconsof RhlI gene from isolates NO 21,22.23,24,25 Lanes from 6 to 10 were amplicons of PelB gene from isolates NO 21,22,23,24,25.

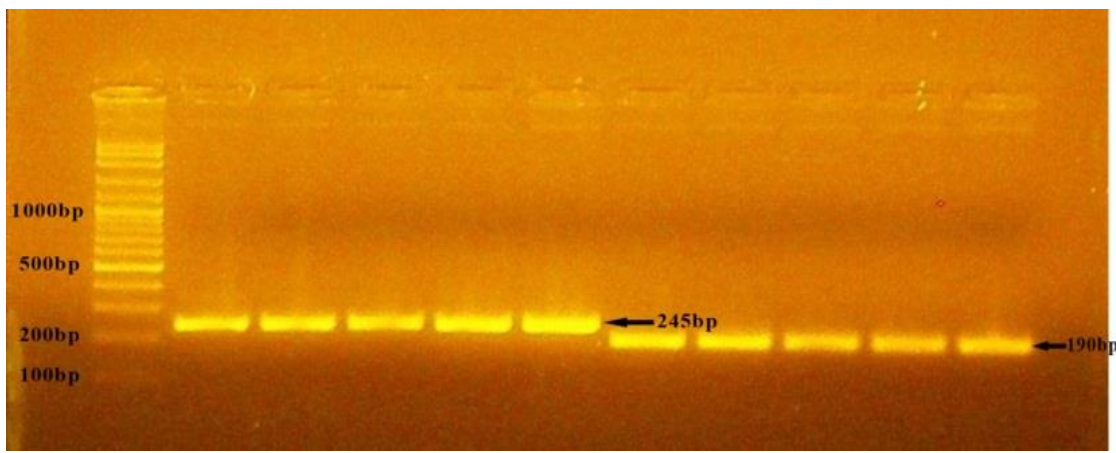

Fig (15) Agarose gel electrophoresis of RhlI gene amplicons(245 bp) andPelB gene amplicons (190 bp). MWM-molecular weight marker (100 bp).Lanes from 1 to 5 were ampliconsof RhlI gene from isolates NO 26,27,28,29,30 Lanes from 6 to 10 were amplicons of PelB gene from isolates NO 26,27,28,29,30. 


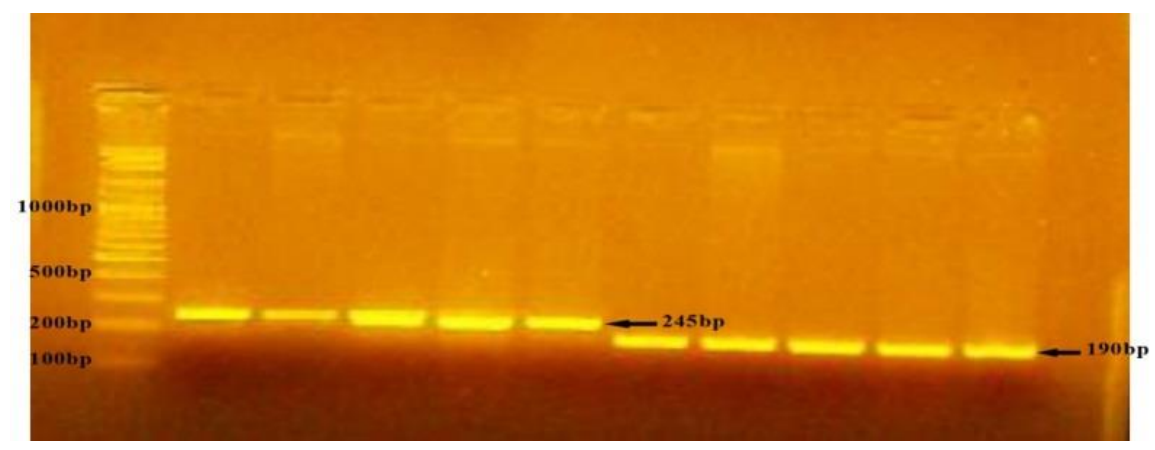

Fig (16) Agarose gel electrophoresis of RhlI gene amplicons(245 bp) andPelB gene amplicons (190 bp). MWM-molecular weight marker (100 bp).Lanes from 1 to 5 were ampliconsof RhlI gene from isolates NO 31,32,33,34,35 Lanes from 6 to 10 were amplicons of PelB gene from isolates NO $31,32,33,34,35$.

\section{Discussion}

Pseudomonas aeruginosa is known as a leading cause of nosocomial infections worldwide. Antimicrobial resistance and biofilm production, as two main virulence factors of $P$. aeruginosa are responsible for the persistence of prolonged infections [14]. Biofilm-forming bacteria are often seen on the surfaces of tissues and biomaterials at sites of persistent infection. Medical implants and catheters are particularly susceptible to biofilm formation because immune responses are significantly reduced in proximity to foreign bodies [15]. It was discovered that $\mathrm{P}$. aeruginosa quorum-sensing (QS) signal molecules termed autoinducers (AI) play a significant role in the differentiation process . P. aeruginosa has two hierarchical QS systems known as las and rhl [4].

In the present study we assessed the role of some selected genes and the formation of biofilm in 35 clinically isolates of drug resistant Pseudomonas aeruginosa obtained from tips of central venous catheters, 19 (54.3\%), urine collected from urine catheters, $8(22.9 \%)$, synthetic vascular grafts, $5(14.3 \%)$ and peripheral catheters, $3(8.6 \%)$.

The results of the present work showed that females 12 (34\%), males $23(65 \%)$. Similar results were revealed by Ullah et al.[16] (62.5\%) of the subjects were males whereas $(37.5 \%)$ were females and Khan et al.[17] (61\%) of the subjects were males whereas $(39.0 \%)$ were females.

The gender-wise prevalence of isolates shows that infections caused by P. aeruginosa are more common in males than females. The reason for high incidence in male might be due to habits of smoking and consumption of alcohol and use of narcotic drugs which has a direct correlation with poor immune system. Frequent hospital visit, use of extensive antibiotics and personal hygiene may also contribute the prevalence of higher bacterial density in males than females [18].

The results of the present work showed that the majority of $\mathrm{P}$. aeruginosa isolates were isolated from haematology unit $(37.1 \%)$. The lower rate $(8 \%)$ was reported by Tacconelli et al.[19].
The results of the present work showed that the lowest percentage of $\mathrm{P}$. aeruginosa isolates were isolated from $(8.6 \%)$ from burn unit. Higher rates $(54.9 \%)$ were reported by Bhatt et al.[20]and $38.63 \%$ by Abdulhaqet al.[21].

The results of the present work showed that the highest susceptibility $(100 \%)$ was shown to colistin followed by Polymyxim B (97.1\%) , and Imipeneme ( $54.3 \%$ ) .

Similar results were revealed by Yekaniet al. [22],Akhi et al.[23] and (Al-Khudhairy and Al-Shammari ,[24]who reported that $100 \%$ P.aeriogenosa isolates were sensitive to colistin .

Another study by Al-Khudhairy and Al-Shammari ,[24]stated that $100 \%$ P. aeruginosa isolates were sensitive to polymyxin $\mathrm{B}$.

Another study by Saderi and Owlia, [25]stated that the lowest susceptibility was shown for polymyxin antimicrobials (90.9\% and $95.5 \%$ respectively, for colistin and polymyxin $\mathrm{B}$ )

Lower sensitive rates to Aboushleib et al.[26]stated that $94 \%$ P. aeruginosa isolates were sensitive to polymyxim B.

Similar results were revealed by Saderi and Owlia .[27]who reported that $(55.7 \%)$ of P. aeruginosa isolates were susceptible to imipenem.

Lower susceptible rates to imipenem (46.1\%) were revealed by Akhi et al., (23) and $41.94 \%$ were reported by Yekaniet al.[22].

The results of the present work showed that all P. aeruginosa isolates were resistant to cefepime (100\%) followed by Gentamicin and Tobramycin (62.9\%).

Similar results were revealed by Mikomangwa et al .[27]who reported that $(93.8 \%)$ of P. aeruginosa isolates were resistant to cefepime and $94.7 \%$ were reported by Banar et al .[28].

Lower resistance rates to cefepime (45.6\%) were reported by Saderi and Owlia, [25], 50\% by Aboushleib et al. [26] and $55.86 \%$ by Yekani et al. [22] .

Similar results were revealed byYekani et al., [22] who reported that $67.41 \%$ of $\mathrm{P}$. aeruginosa isolates were resistant 
to gentamicin , 63.2\% were reported by Angadi et al. [29] and $66.7 \%$ were reported byDas et.al.[30].

Lower resistance rates to gentamicin $(54 \%)$ were revealed by Aboushleib et al., [26] and $18.75 \%$ were reported by Kamali et al.[14].

Lower resistance rates to Tobramycin $(45.6 \%)$ were revealed by Saderi and Owlia , [25] and $16.25 \%$ were detected by Kamali et al., [14].

This variation in resistance rates among the different studies could be explained by the fact that $\mathrm{P}$. aeruginosa resistance patterns differ internationally, regionally and locally in developed countries due to inappropriate initial antimicrobial therapy ,early interruption of treatment, subtherapeutic doses and also as a result of the overuse of antibiotics [31].

The results of the present work showed that among the 35 studied P. aeruginosa isolates, XDR was detected in 19 isolates $(54.3 \%)$, while 3 isolates $(8.6 \%)$ were considered as MDR and 13 isolates (37.1\%) were Non - MDR/ XDR .

This was in agreement with results were reported by Mirzaei et al. [32] (59.54\% were XDR). On the other hand, lower results were releaved by Saderi and Owlia ,[25](33\% wereXDR) and Samad et al., [33](25\% were XDR)

This was in agreement with results were reported by Morales et al.[34] (5.46\% were MDR), Walkty et al. [35] (6.5\% were MDRandTacconelli et al. [19] (14\% were MDR). On the other hand, higher results were releaved by Salimi et al .[ 36](33.1\% were MDR),Abdulhaq et al, [21] (38.46\% were MDR), Lima et al.[37] (53.3\% were MDR) ,Ghadaksaz et al. [38] (58.68\% were MDR) and Yekani et al. [22] (65\% were MDR)

This was in agreement with results (40.4\%) were reported by Elmaraghy et al .[39]. On the other hand, higher results were releaved by Gill et al.[40] (47.7\% were non MDR- XDR) and Lima et al.[37] (46.7\% were non MDRXDR)

The reasons behind such a resistance are the inappropriate or irrational use of antibiotics, mutation in the genome of P. aeruginosa and environmental conditions of the specific area. [21].

The results of the present work showed that biofilm production by CRA method was as follows: $(68.6 \%)$ of isolated organisms were biofilm producers,

The lower results $(44.23 \%)$ were releaved by Abdulhaq et al .[21] and 9\% were reported by Fattouh et al.[41].

The results of the present work showed that biofilm production by CRA method was as follows: $(31.4 \%)$ were non biofilm producers. The higher rate $(91 \%)$ was reported byFattouh et al.[41].

Inthe current study, according to the measured OD 570 for each isolate, adherence capability of each isolate was classified into three categories : weak, moderate and strong adherent cells according toMathur et al.[11].

Weak biofilm - forming isolates in our study, were found to be $22.9 \%$ of the total isolates tested. This was in agreement with results were reported byAboushleib et al.[26] (24\%) and Fattouh et al.[41] (22\%). Higher results $(33.75 \%)$ were reported by Kamali et al.[14]and $40 \%$ were detected byLima JL et al. [37] while lower results (17.3\%) were reported by Abdulhaq et al. [21], 10\% were reported by Hisham et al.[42] and $8 \%$ were detected by Elhabibi and Ramzy,[43].

As regard the moderate biofilm - forming isolates, in this work, they represented $31.4 \%$ among all isolates tested . Similar results (25\%) were reported by Lima et al.[37] .Lower results (19\%) were reported by Fattouh et al.[41] and $10 \%$ were detected by Hisham et al.[42].

As regard the strong biofilm - forming isolates, in this work, they represented $45.7 \%$ among all isolates tested . Similar results $(36.5 \%)$ were reported by Abdulhaq et al. [21]. Higher results $(80 \%)$ were reported by Hisham et al.[42] and $84 \%$ were detected by Abd El-Galil et al.[44].While lower results (10\%) were reported by Lima et al.[37] and 17\% were detected by Fattouh et al.[41].

On the basis of Congo red agar test 24 (68.6\%) samples showed biofilm forming potential while when the Tissue Culture Plate method (TCP) was applied 35(100 \%) samples showed variable degree of biofilm production. Highest percentage was found $(45.7 \%)$ of strong biofilm producing Pseudomonas aeruginosa followed by moderate biofilm producers $(31.4 \%)$.

This variation in the rate of biofilm grading among the different studies may be due to the difference in the number of clinical isolates from different sources [45].

The results of the present work showed that the relation between biofilm grading and antibiotic resistance, it was observed that the moderate biofilm - forming isolates were XDR $(31.6 \%)$ compared to the strong biofilm forming $(52.6 \%)$

Where as the moderate biofilm forming isolates were MDR (66.7\% )compared to the strong biofilm forming( $33.3 \%$ ) . Similar results were reported by Abdulhaq et al.[21] the moderate biofilm forming isolates were MDR (52.3\%) compared to the strong biofilm forming $(42.1 \%)$

The discrepancy of the previous results can be attributed to the different mechanisms that may cause resistance to antibiotics .Resistance patterns amongst hospital - acquired bacterial pathoges are often different from country to country and within a single country over time [46].

In the present study, it was found that the two selected genes(RhlI- PelB) were detected in both strong biofilm producer isolates and weak biofilm producer isolates suggesting that these genes are contributing in biofilm formation in P. aeruginosa as they were detected in the strong biofilm producers isolates.

Also, we suggest that there are other factors contributing to biofilm formation in P. aeruginosa as these genes were detected also in weak biofilm producer isolates. These factors may be strain difference, culture conditions, rate of expression of these genes, presence of other genes needed for biofilm formation or other factors affecting biofilm formation. 


\section{Conclusions}

From the present study it can be concluded that:Biofilm formation is highly prevalence among the clinical isolates of Pseudomonas aeruginosa in Benha University Hospital and Benha Children Hospital which could potentially increase colonization of antibiotic- resistant bacteria in hospital environments.

This study indicated that there were a significant percentage of organisms able to grow within biofilm produced on indwelling medical device surfaces and considered as a source of infections. The rate of biofilm formation on catheter was found to increasing duration of catheter insertion Biofilms cause resistance to many antimicrobial agents. The results of biofilm produced on indwelling medical devices are recurrent, untreatable infections and failure of medical device.

The XDR Pseudomonas aeruginosa isolates are more prevalent than MDR clinical isolates. All biofilm-forming isolates are resistant to cefepime. In addition, they are more frequently resistant to Gentamicin and Tobramycin.

Colistin proved to be a highly active antimicrobial agent against Pseudomonas spp. Hence, it is an important alternative treatment option and may be considered as empiric therapy in serious infections when these organisms are suspected and the resistance rates are high in the hospital setting.

The strong biofilm- forming catogry is more prevailing among MDR clinical isolates, while the moderate biofilm - forming category is the predominant one among XDR clinical isolates. the present study, it was found that the two selected genes(RhlI- PelB) were detected in both strong biofilm producer isolates and weak biofilm producer isolates suggesting that these genes are contributing in biofilm formation in $P$. aeruginosa as they were detected in the strong biofilm producers isolates.

Also, we suggest that there are other factors contributing to biofilm formation in $P$. aeruginosa as these genes were detected also in weak biofilm producer isolates. These factors may be strain difference, culture conditions, rate of expression of these genes, presence of other genes needed for biofilm formation or other factors affecting biofilm formation.

\section{Referances}

[1] K.Streeter ,M.Katouli , Pseudomonas aeruginosa: A review of their Pathogenesis and Prevalence in Clinical Settings and the Environment. Infect Epidemiol Med. Winter, Vol. Volume 2, Issue 1, PP. 25-32,2016.

[2] K.Zhang, X.Li, Y.u. Chen and Y.Wang,Promising Therapeutic Strategies Against Microbial Biofilm Challenges.Frontiers in Cellular and Infection Microbiology, Vol.10, PP.359, 2020.

[3] J. N. Anderl, M. J. Franklin and P. S. Stewart, Role of antibiotic penetration limitation in Klebsiella pneumoniae biofilm resistance to ampicillin and ciprofloxacin. Antimicrob Agents Chemother, Vol.44(7), PP.1818-24,2000.

[4] M.Schuster, E. P. Greenberg ,A network of networks: quorumsensing gene regulation in Pseudomonas aeruginosa. Int.J. Med. Microbiol, PP. 296, 2006.

[5] E. E. Mann and D. J. Wozniak,Pseudomonas biofilm matrix composition and niche biology. FEMS Microbiol Rev, Vol.36(4), PP.893-916. 2012

[6] S.Marmont, D.Rich, C.Whitney,Oligomeric lipoprotein PelC guides Pel polysaccharide export across the outer membrane of Pseudomonas aeruginosa. PNAS, Vol.114(11), PP.2893-2897,2017.

[7] UK Standards for Microbiology Investigations ,Identification of Pseudomonas species and other NonGlucose Fermenters. Bacteriology - Identification;ID , Vol.17(3), PP.1-41,2015.

[8] Clinical and Laboratory Standards Institute , Zone diameter and minimal inhibitory concentration interpretive standards for Pseudomonas.M100-S25.Clinical and laboratory Standards Institute, Wayne, PA, USA, 2016.

[9] Clinical and Laboratory Standards Institute ,Performance Standards for Antimicrobial Susceptibility Testing. 30th ed. CLSI supplement M100. Wayne, PA: USA,2020.

[10] Magiorakos, A.Srinivasan, R.Carey, Multidrugresistant, extensively drug-resistant and pandrugresistant bacteria: an international expert proposal for interim standard definitions for acquired resistance. Clin Microbiol Infect, Vol.18(3), PP. 268-281,2011.

[11] T.Mathur, S.Singhal, S.Khan,Detection of biofilm formation among the clinical isolates of staphylococci: an evaluation of three different screening methods. Indian J Med Microbiol, Vol. 24(1), PP.25-9,2006.

[12] D. J.Freeman, F. R. Falkiner and C. T. Keane ,New method for detecting slime production by coagulase negative staphylococci. J Clin Pathol, Vol.42(8), PP.872-874,1989.

[13] A.Jain ,A.Agarwal , Biofilm production, a marker of pathogenic potential of colonizingand commensal Staphylococci.J Microbiol Methods, Vol.76(1), PP.8892,2009.

[14] E.Kamali, A.Jamali and A.Ardebili ,Evaluation of antimicrobial resistance, biofilm forming potential, and the presence of biofilm-related genes among clinical isolates of Pseudomonas aeruginosa. BMC Res Notes, Vol.13, PP.27, 2020.

[15] K.Seifi, H.Kazemian, H.Heidari,Evaluation of Biofilm Formation Among Klebsiella pneumoniae Isolates and Molecular Characterization by ERIC-PCR, Jundishapur J Microbiol, Vol.9(1), PP.e30682,2016.

[16] N.Ullah, E.Güler, M.Güvenir3,Isolation, Identification, and Antibiotic Susceptibility Patterns of Pseudomonas aeruginosa Strains from Various Clinical Samples in a University Hospital in Northern Cyprus ,Cyprus J Med Sci , Vol. 4(3) , PP.225-8,2019. 
[17] J.Khan, A.Wahab, A.Qayyum ,Drug resistance pattern of Pseudomonas aeruginosa isolates at PIMS Hospital, Islamabad, Pakistan , J.,Chemical and Pharmaceutical Research, Vol. 6(11), PP.715-719,2014.

[18] R.Pradeepraj, D. Jayarajan and M.Harish 'Biofilm and Mettalo Beta-Lactamase Production in Association With Serum Resistant Activity Among Clinical Strains of Pseudomonas aeruginosa A Web of Science - ESCI Indexed Journal, 2019.

[19] E.Tacconelli, M.Tumbarello, S.Bertagnolio, Multidrug-resistant Pseudomonas aeruginosa bloodstream infections: analysis of trends in prevalence and epidemiology. Emerg Infect Dis, Vol.8(2), PP.220-1,2002.

[20] P.Bhatt, K. R. Rathi, S.Hazra,Prevalence of multidrug resistant Pseudomonas aeruginosa infection in burn patients at a tertiary care centre . Indian J Burns 2015, Vol.23, PP.56-9,2015.

[21] N.Abdulhaq, Z.Nawaz, M. A. Zahoor, Association of Biofilm Formation With Multi Drug Resistance in Clinical Isolates of Pseudomonas aeruginosa,EXCLI Journal, Vol.19, PP.201-208 - ISSN 1611-2156,2020.

[22] M.Yekani, M. Y.Memar , N.Alizadeh,Antibiotic Resistance Patterns of Biofilm-Forming Pseudomonas Aeruginosa Isolates from Mechanically Ventilated Patients International J.,Scientific Study, Vol. 5 |, PP.Issue 5 ,2017.

[23] M. T. Akhi, R.Ghotaslou, S.Beheshtirouy,Antibiotic susceptibility pattern of aerobic and anaerobic bacteria isolated from surgical site infection of hospitalized patients. Jundishapur J.,microbiology, PP.8(7),2015.

[24] M. K . Al-Khudhairy , M.Al-Shammari ,Prevalence of metallo--lactamase Producing Pseudomonas aeruginosa isolated from diabetic foot infections in Iraq. -New Microbes and New Infections, Vol. Number C,2020.

[25] H. Saderi ,P. Owlia ,Detection of Multidrug Resistant (MDR) and Extremely Drug Resistant (XDR) Pseudomonas AeruginosaIsolated from Patients inTehran,Iran, Fall IRANIAN J.,PATHOLOGY, Vol.10, PP.4,2015.

[26] H. M.Aboushleib, H. M.Omar, R.Abozahra, Correlation of quorum sensing and virulence factors in Pseudomonas aeruginosa isolates in Egypt $\mathrm{J}$ Infect Dev Ctries, Vol. 9(10), PP.1091-1099,2015.

[27] W. P.Mikomangwa, G. M. Bwire, M. Kilonzi, The Existence of High Bacterial Resistance to Some Reserved Antibiotics in Tertiary Hospitals in Tanzania: A Call to Revisit Their Use nfection and Drug Resistance, Vol.13, PP.1831- 1838, 2020.

[28] M.Banar, M.Emaneini, M.Satarzadeh, Evaluation of mannosidase and trypsin enzymes effectson biofilm production of Pseudomonas aeruginosa isolated from burn wound infections. PLoS ONE. vol. 11(10), PP. e0164622, 2016.
[29] K.M. Angadi , M.Kadam ,M.S. Modak, Detection of Antibiotic Resistance In Pseudomonas aeruginosa Isolates with special Referance to Metallo B Lactamas from a Tertiary Care Hospital in Western India international J.,Microbiology Research ISSN: 0975 -5276, E -ISSN: 0975 -9174, Vol.4, PP. Issue 7, 2012.

[30] S.Das, M.Azad, K.Bimal,Antimicrobial Susceptibility Pattern of Pseudomonas aeruginosa with Special Reference to ESBL Producers from Various Clinical Samples at aTertiary Care Center in Bihar International J.,Research and Review, Vol.7; Issue, PP. 1,2020.

[31] L.Farooq, Z.Memon and M.Owais ,Frequency and antibiogram of multi-drug resistant pseudomonas aeruginosa in a Tertiary Care Hospital of PakistanPak $\mathbf{J}$ Med Sci November - December , Vol. 35 No. 6 , PP.1622-1626, 2019.

[32] B.Mirzaei, Z. N. Bazgir, H. R. Goli,Prevalence of multi-drug resistant (MDR) and extensively drugresistant (XDR) phenotypes of Pseudomonas aeruginosa and Acinetobacter baumannii isolated in clinical samples from Northeast of Iran BMC Res Notes , Vol.13, PP.380,2020.

[33] A.Samad, T.Ahmed, A.Rahim,Antimicrobial susceptibility patterns of clinical isolates of Pseudomonas aeruginosa isolated from patients of respiratory tract infections in a Tertiary Care Hospital, Peshawar. Pak J Med Sci, Vol.33, PP.670-4,2017.

[34] E.Morales, F.Cots, M.Sala, Hospital costs of nosocomial multi-drug resistant Pseudomonas aeruginosa acquisition. BMC Health ServRes, Vol.12, pp. $122,2012$.

[35] A.Walkty , J. A.Karlowsky , H.Adam , In vitro activity of ceftolozane- tazobactam against Pseudomonas aeruginosa isolates obtained from patients in Canadian hospitals in the CANWARD study, 2007 to 2012. Antimicrob Agents Chemother, Vol.57(11), PP.5707-5709,2013.

[36] H.Salimi, B.Yakhchali, P.Owlia, MolecularEpidemiology and Drug Susceptibility ofPseudomonas aeruginosa Strains Isolated from BurnPatients. LabMedicine, Vol.41 (9), PP.5404,2010.

[37] J. L. Lima, L. R. Alves, J. N.Paz , Prevalence and susceptibility pattern of multi drug resistant clinical isolates of Pseudomonas aeruginosa in Karachi. Pak J Med Sci, Vol.30(5), PP.951-954,2017.

[38] A.Ghadaksaz, A. A.Fooladi, H. M.Hosseini,The prevalence of some Pseudomonas virulence genes related to biofilm formation and alginate production among clinical isolates. J.,Applied Biomedicine. Vol. 13(1), PP.61-8,2015.

[39] N.Elmaraghy, S.Abbadi, G.Elhadidi, Virulence Genes in Pseudomonas Aeruginosa Strains Isolated at Suez Canal University Hospitals with Respect to the Site of 
Infection and Antimicrobial Resistance. Int $\mathrm{J}$ Clin Microbiol Biochem Technol, Vol. 2, PP.008-01,2019.

[40] S. J.Gill, S.Arora, S. P.Khanna,Prevalence of Multidrug-resistant, Extensively Drug-resistant, and Pandrug-resistant Pseudomonas aeruginosa from a Tertiary Level Intensive Care UnitOct-Dec, Vol. 8(4), PP. 155-15 J.,Global Infectious Diseases, 2016.

[41] M.Fattouh, A.Nasr El-din, N.Ahmed,Inhibition of Quorum Sensing Character in Pseudomonas aeruginosa isolates and Its Effect on Biofilm Formation and AntiMicrobial Susceptibility Profile Egyptian J.,Medical Microbiology, Vol. 27 / , PP. 25-33,2018.

[42] A Hisham, M. S. Fathy and M. E. Eman ,Combating Pseudomonas aeruginosaBiofilms by Potential Biofilm Inhibitors. Asian J Res Pharm Sci, Vol.2, PP. 66-72, 2012.

[43] T.Elhabibi ,S. Ramzy ,Biofilm Production by Multi Drug Resistant Bacterial Pathogens Isolated From
Patients in Intensive Care Units in Egyptian Hospitals J Microb Biochem Technol, Vol. 9, PP.151-158, 2017.

[44] K.Abd El Galil, S. M Abdel Ghani, M. A. Sebak, Detection ofbiofilm genes among clinical isolates of Pseudomonas aeruginosa recoveredfrom some Egyptian hospitals. N Egypt J Microbiol, PP. 36,2013.

[45] A.Abdi-

Ali,S.Hendiani,P.Mohammadi,Assessmentofbiofilmfor mation andresistancetoimipenemandciprofloxacin among clinicalisolatesofAcinetobacter baumannii in Tehran. Jundishapur J. Microbiol, Vol.7(1), PP.e8,2014.

[46] A.deBreij,J.Gaddy,J.vanderMeer,CsuA/BABCDEdependentpiliarenot involvedintheadherenceofAcinetobacterbaumanniiATC C19606(T) tohumanairway epithelial cells andtheirinflammatoryresponse.Res. Microbiol, Vol. 160, PP. 213-218,2009. 